\title{
Focusing of light by a nanohole array
}

\author{
Fu Min Huang ${ }^{\text {a) }}$ and Nikolay Zheludev \\ Optoelectronics Research Center, University of Southampton, Southhampton SO17 1BJ, United Kingdom \\ Yifang Chen \\ Central Microstructure Facility, Rutherford Appleton Laboratory, Didcot OX11 OQX, United Kingdom
}

F. Javier Garcia de Abajo

Instituto de Optica, CSIC, Serrano 121, 28006 Madrid, Spain

(Received 16 November 2006; accepted 27 January 2007; published online 2 March 2007)

\begin{abstract}
The authors demonstrate a mechanism for focusing at optical frequencies based on the use of nanohole quasiperiodic arrays in metal screens. Using coherent illumination at $660 \mathrm{~nm}$ and scanning aperture optical microscopy, $\sim 290 \mathrm{~nm}$ "hot spots" were observed at a distance of $\sim 12.5 \mu \mathrm{m}$ from the array. Even smaller hot spots of about $\sim 200 \mathrm{~nm}$ in waist were observed closer to the plane of the array. () 2007 American Institute of Physics. [DOI: 10.1063/1.2710775]
\end{abstract}

Nanoholes and arrays of nanoholes in metal screens show a number of intriguing optical properties, most notably the extraordinarily high transmission of regular $^{1}$ and quasiperiodic ${ }^{2}$ hole arrays and unusual polarization-sensitive effects. ${ }^{3,4}$ Here we show that a quasicrystal array of nanoholes in a metal screen can "focus" light at distances up to several tens of wavelengths from the screen. It produces bright foci of energy concentration, harvesting energy from a large number of holes in the array. The foci are sparsely distributed in the "focal plane."

The "focusing" of light was observed with a quasicrystal array illuminated with a coherent light source. The array had an approximate tenfold symmetry and contained about 14000 holes of $200 \mathrm{~nm}$ diameter. The overall diameter of the array was $\sim 0.2 \mathrm{~mm}$. The array comprised a Penrose-like pattern constructed in accordance with the algorithm described in Ref. 5. It was manufactured by electron beam lithography in a $100 \mathrm{~nm}$ aluminum film deposited on a silica substrate [see Fig. 1(a)]. The high level of positional accuracy achieved in the manufacturing of the holes may be judged from a comparison of the far-field diffraction pattern produced by the array and the reciprocal lattice, calculated from the prescribed coordinates of the holes. Allowing for expected axial distortion and scaling these should be congruent mappings of each other, and indeed they are [compare Figs. $1(\mathrm{~b})$ and $1(\mathrm{c})]$.

To map the field distributions created by the array we used a scanning aperture optical microscope (Omicron TwinSNOM R/T) operating in transmission mode, with light collected through a metal-coated tapered fiber tip with an aperture of about $200 \mathrm{~nm}$ (Fig. 2) or $120 \mathrm{~nm}$ (Fig. 3). As for optical sources we used either diode lasers operating at 660 and $635 \mathrm{~nm}$ or a continuum fiber laser (with a bandpass filter centered at $500 \mathrm{~nm}$, bandwidth of $40 \mathrm{~nm}$ ). Figure 2 shows characteristic field maps measured at different heights $h$ above the plane of the array for two different wavelengths, thus illustrating the variety of "photonic carpet" patterns generated by the quasicrystal structure. In most cases, the carpets show elements of approximate fivefold or tenfold symmetry. In the immediate proximity of the sample [Figs. 2(a) and 2(b)], the optical field concentrates at the holes.

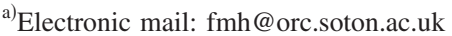

Further away from the array, the pattern changes dramatically and becomes blurry and defocused [Figs. 2(e) and 2(f)]. This alternates with well-defined arrangements of hot spots [Figs. 2(c), 2(d), 2(g), and 2(h)] that appear at specific, irregular heights. At some heights and wavelengths bright foci, separated by a few microns from the neighboring spots, are seen [Figs. 2(g) and 2(h)]. The patterns depend not only on the wavelength and height but also on the distance between the focus of the illuminating lens and the quasicrystal structure.

Figure 3 shows a detailed scan of the foci at two characteristic distances, with the array illuminated at a wavelength of $660 \mathrm{~nm}$. At a distance of $\sim 5 \mu \mathrm{m}$ from the array, a relatively dense pattern of hot spots is seen. Here the bright foci are elliptical with a full width at half maximum of
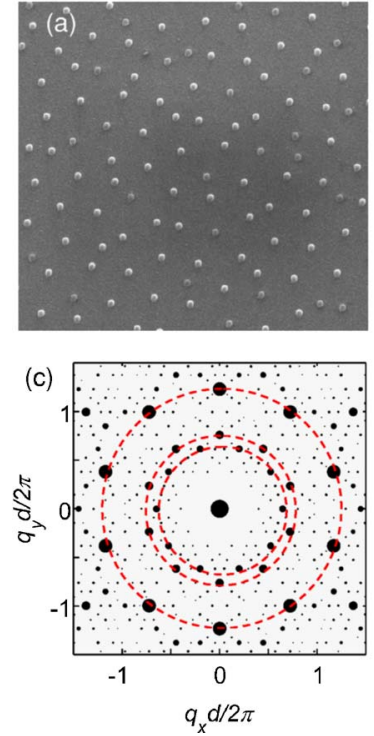

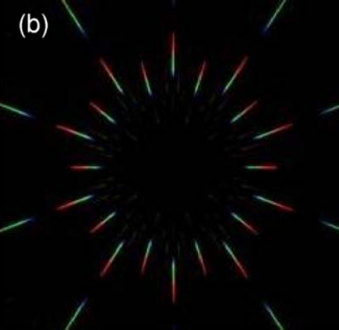

(d)

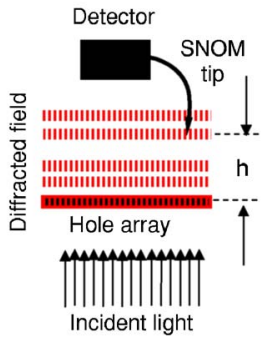

FIG. 1. (Color online) Quasicrystal sample and its reciprocal lattice. (a) Scanning electron microscopy image of a fragment $\left(20 \times 20 \mu \mathrm{m}^{2}\right)$ of the quasicrystal array of holes. (b) Far-field diffraction pattern of the quasicrystal array, obtained with white light illumination. (c) Reciprocal lattice of the quasicrystal array wherein spot diameter is proportional to the magnitude of the spectral component. The minimum distance between two neighboring holes $d=1.2 \mu \mathrm{m}$. Red dashed circles show several "partial" Montgomery rings. (d) Schematic of the experiment showing field pattern over the array illuminated with coherent light. 

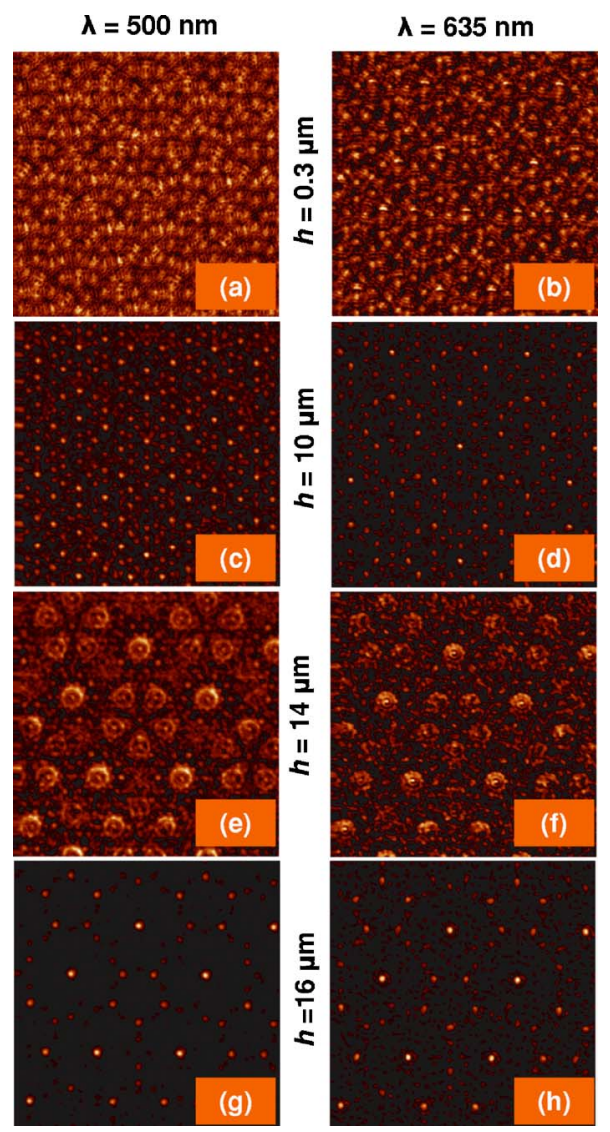

FIG. 2. (Color online) Field maps at different heights $h$ above the quasicrystal array of holes. Each scan is $30 \times 30 \mu \mathrm{m}^{2}$ in area. The images were obtained by SNOM using illumination wavelengths of $500 \mathrm{~nm}$ (left) and $635 \mathrm{~nm}$ (right). Note the highly isolated hot spots observed at $h=16 \mu \mathrm{m}$.

$\sim 375 \mathrm{~nm}$ along the polarization direction of the incident light and $\sim 235 \mathrm{~nm}$ in the perpendicular direction. After deconvolution to account for the aperture size of the SNOM probe using Gaussian approximation for the tip field profile, the actual size of the hot spots is estimated to be about $360 \times 200 \mathrm{~nm}^{2}(0.54 \lambda \times 0.30 \lambda)$. A much clearer pattern of well-isolated hot spots is seen at a distance of $\sim 12.5 \mu \mathrm{m}$ from the array. Here the scanned cross-sectional dimensions are $420 \times 320 \mathrm{~nm}^{2}$, and after deconvolution the spot size obtained is about $400 \times 290 \mathrm{~nm}^{2}(0.60 \lambda \times 0.44 \lambda)$. Thus, the structure produces bright foci of energy concentration by harvesting energy from a large number of holes. For example, in Figs. 2(g) and 2(h) the density of the brightest hot spots (arranged in pentagons), which receive the majority of the energy, is some 40 times lower than that of the holes in the array.

This new phenomenon of "lensing" by the nanohole array results from the partial reconstruction of the array's field in the diffraction zone, in a manner analogous to the classical Talbot effect ${ }^{6}$ observed with periodic gratings, where diffraction leads to the reconstruction of the grating's field at periodic distances from the grating. In the Talbot effect a grating with a period $a$ images itself at multiples of the Talbot distance $T=a^{2} / \lambda$ when illuminated with a coherent plane wave. In the paraxial approximation, an infinitely long grating will be perfectly reconstructed at the Talbot distance.

Montgomery ${ }^{7}$ has shown that a wide range of patterns can image themselves, with linear periodic grating being only one example. A pattern will show full reconstruction at Downloaded 05 Mar 2007 to 152.78.194.72. Redistribution subject
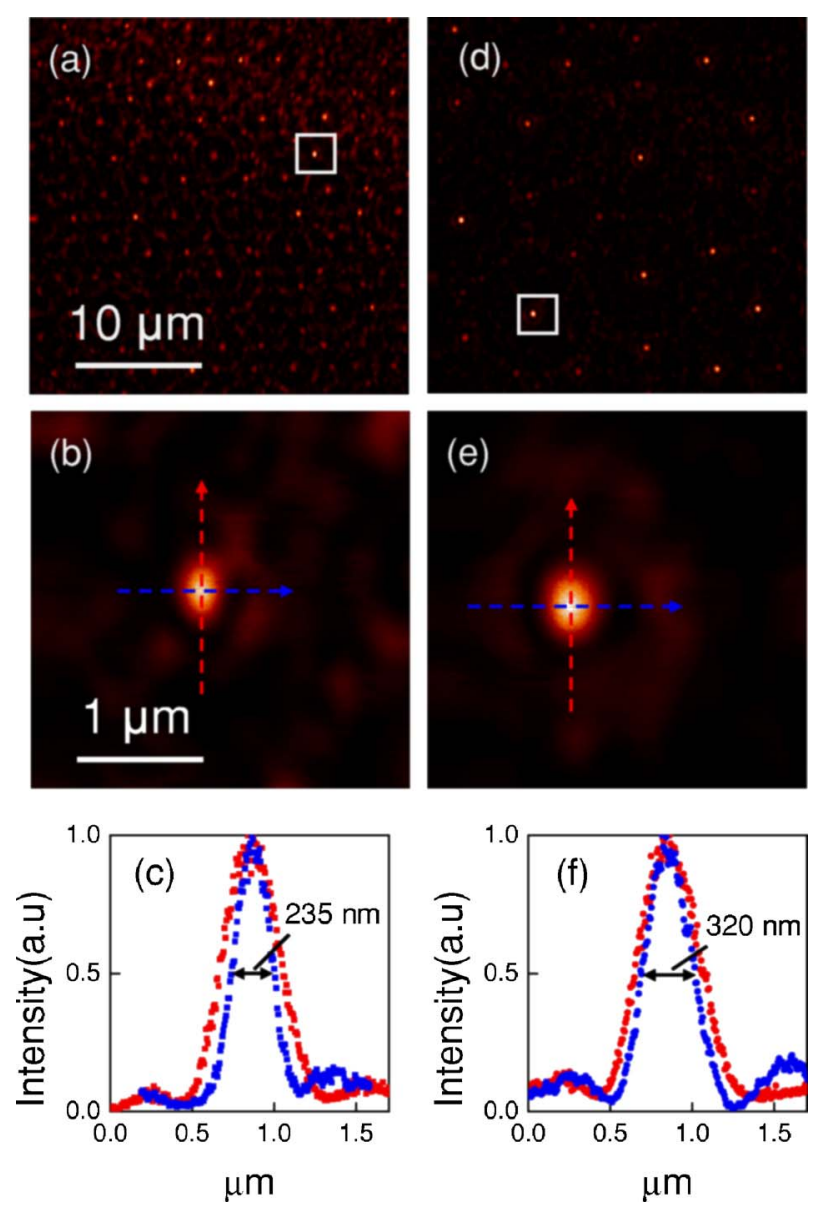

FIG. 3. (Color online) Subwavelength hot spots formed at different heights above the quasicrystal array. The illumination source is a diode laser with a wavelength of $660 \mathrm{~nm}$. (a) Field map at a height $h=5 \mu \mathrm{m}$ above the array. [(b) and (c)] Fine scan image of the hot spot indicated in map (a) and corresponding profiles of the focus along directions parallel (blue dots) and perpendicular (red dots) to the polarization of the incident light. (d) Field map at a height $h=12.5 \mu \mathrm{m}$ above the array. [(e) and (f)] Fine scan image of the hot spot indicated in map (d) and corresponding profiles of the focus along directions parallel (blue dots) and perpendicular (red dots) to the polarization of the incident light.

a distance $h$ if its spatial frequencies are discrete and located in the reciprocal plane at rings of radii $\rho^{2}=1 / \lambda^{2}-(m / h)^{2}$, where $m$ is an integer such that $0 \leqslant m \leqslant h / \lambda$. For instance, linear diffraction gratings are self-imaging objects. As the rich spectrum of frequencies of the quasiperiodic array are located on circles [as shown in Fig. 1(b)] the Montgomery condition is fulfilled for all spectral maxima but not necessarily simultaneously. Therefore, the self-imaging distance $h$ will be different for different rings of maxima on the reciprocal lattice and for different wavelengths. Moreover, when a regular grating is illuminated with a divergent beam, the reconstructed image is magnified, so the reconstructed field does not necessarily have the same period as the original grating. Similarly, when partial reconstruction of the quasiperiodic array takes place, the pattern of reconstructed hot spots has the appearance of a scaled partial image of the array. Thus, reconstruction of a quasiperiodic array of holes is a complex process which may be envisaged as a superposition of a large number of partial reconstructions happening at different heights at the array. With varying height $h$, one will see a continuous evolution of partially reconstructed images of the array. What is important, however, is that at some distances and wavelengths well-defined sparsely distributed o AlP license or copyright, see http://apl.aip.org/apl/copyright.jsp 


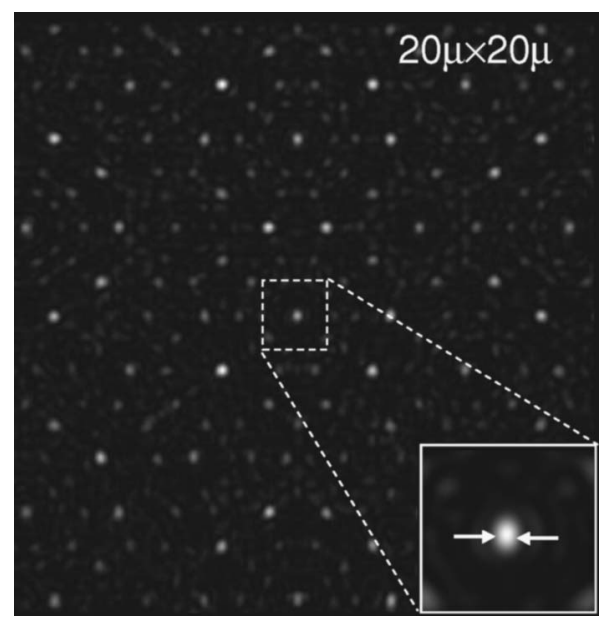

FIG. 4. Theoretical calculations at the conditions close to that of Fig. 2(c) show a pattern similar to that observed experimentally and give hot spots of $220 \mathrm{~nm}$ in cross section.

foci are seen. The subwavelength nature of this spots may be relevant to the effect of achieving rapid changes of a field known as superoscillation. ${ }^{8}$ To illustrate the process of quasiperiodic grating reconstruction, we have calculated the field intensity maps created by quasicrystal array using the scalar diffraction theory without taking into account evanescent fields. The calculation gives very good agreement with experimental results as illustrated by Fig. 4.

The subwavelength focusing of light by a nanohole array could provide numerous applications in nano-optics. For instance, a single hot spot appropriately isolated by a mask may be used as a light pen for subwavelength imaging by scanning the object under investigation across the focal spot. Its advantage over the conventional near-field probe is in the large distance between the nanohole array focus and the object, which is in the order of a few microns. Therefore the array may be used for focusing inside cells and other microscale objects. The considerable gap between the array and the object will allow much faster scanning and rapid image acquisition than are achievable by near field optical microscopy. ${ }^{9}$ As the focus harvests light from a large number of holes in the array, a higher level of optical intensity may be delivered to the hot spot in comparison with a single aperture device. With such high localization of optical energy and subwavelength resolution, other applications can also be envisaged, such as high resolution photolithography, ${ }^{10}$ high-density optical information storage, ${ }^{11}$ and optical trapping. ${ }^{12}$

In conclusion, the focusing of coherent light by a quasicrystal array of holes has been observed at distances ranging from a few microns to a few tens of microns from the plane of the array. With focused hot spots of such high localization and brightness, resolution exceeding that of sophisticated high-numerical aperture lenses imaging can be achieved, which may find important applications in a wide range of areas.

This work was supported by the EPSRC. The authors thank Nikitas Papasimakis and Alexander Schwaneke for taking far-field diffraction patterns of the array and Kevin Macdonald for help in preparing the letter.

${ }^{1}$ T. W. Ebbesen, H. J. Lezec, H. F. Ghaemi, T. Thio, and P. A. Wolff, Nature (London) 391, 667 (1998).

${ }^{2}$ A. S. Schwanecke, N. Papasimakis, V. A. Fedotov, F. Huang, Y. Chen, F. J. García de Abajo, and N. I. Zheludev, presented at the Nanophotonics Topical Meeting (NANO) at IPRA/NANO OSA Collocated Topical Meetings, Uncasville, CT, 24-28 April 2006 (unpublished); F. Preybilla, C. Genet, T. W. Ebbesen, Appl. Phys. Lett. 89, 121115 (2006).

${ }^{3}$ A. V. Krasavin, A. S. Schwanecke, M. Reichelt, T. Stroucken, S. W. Koch, E. M. Wright, and N. I. Zheludev, Appl. Phys. Lett. 86, 201105 (2005).

${ }^{4}$ J. Elliott, I. I. Smolyaninov, N. I. Zheludev, and A. V. Zayats, Phys. Rev. B 70, 233403 (2004).

${ }^{5}$ E. A. Lord, K. Ramakrishnan, and S. Ranganathan, Bull. Mater. Sci. 23, 119 (2000).

${ }^{6}$ K. Patorski, Prog. Opt. 27, 3 (1989).

${ }^{7}$ W. D. Montgomery, J. Opt. Soc. Am. 57, 772 (1967).

${ }^{8}$ M. V. Berry and S. Popescu, J. Phys. A 39, 6965 (2006).

${ }^{9}$ E. Betzig, P. L. Finn, and J. S. Weiner, Appl. Phys. Lett. 60, 2484 (1992).

${ }^{10}$ H. Schmid, H. Biebuyck, B. Michek, and O. J. F. Martin, Appl. Phys. Lett. 72, 2379 (1998).

${ }^{11}$ I. J. Cox, Appl. Opt. 23, 3260 (1984).

${ }^{12}$ A. Ashkin, J. M. Dziedzic, J. E. Bjorkholm, and Steven Chu, Opt. Lett. 11, 288 (1986). 